\title{
Genomic dissection of a 'Fuji' apple cultivar: re-sequencing, SNP marker development, definition of haplotypes, and QTL detection
}

\author{
Miyuki Kunihisa*1), Shigeki Moriya ${ }^{2)}$, Kazuyuki Abe ${ }^{2)}$, Kazuma Okada ${ }^{2)}$, Takashi Haji²), Takeshi Hayashi ${ }^{3)}$, \\ Yoshihiro Kawahara', Ryutaro Itoh ${ }^{4,5)}$, Takeshi Itoh ${ }^{4)}$, Yuichi Katayose ${ }^{4)}$, Hiroyuki Kanamori ${ }^{4)}$, \\ Toshimi Matsumoto ${ }^{4)}$, Satomi Mori ${ }^{4)}$, Harumi Sasaki ${ }^{4)}$, Takashi Matsumoto ${ }^{4)}$, Chikako Nishitani ${ }^{1}$, \\ Shingo Terakami ${ }^{1)}$ and Toshiya Yamamoto ${ }^{1)}$
}

1) NARO Institute of Fruit Tree Science, 2-1 Fujimoto, Tsukuba, Ibaraki 305-8605, Japan

2) NARO Institute of Fruit Tree Science, 92-24 Shimokuriyagawa, Morioka, Iwate 020-0123, Japan

3) NARO Agricultural Research Center, 3-1-1 Kannondai, Tsukuba, Ibaraki 305-8666, Japan

4) National Institute of Agrobiological Sciences, 2-1-2 Kannondai, Tsukuba, Ibaraki 305-8602, Japan

5) DYNACOM Co., Ltd., E-25F, WBG, 2-6-1 Nakase, Mihama-ku, Chiba 261-7125, Japan

'Fuji' is one of the most popular and highly-produced apple cultivars worldwide, and has been frequently used in breeding programs. The development of genotypic markers for the preferable phenotypes of 'Fuji' is required. Here, we aimed to define the haplotypes of 'Fuji' and find associations between haplotypes and phenotypes of five traits (harvest day, fruit weight, acidity, degree of watercore, and flesh mealiness) by using 115 accessions related to 'Fuji'. Through the re-sequencing of 'Fuji' genome, total of 2,820,759 variants, including single nucleotide polymorphisms (SNPs) and insertions or deletions (indels) were detected between 'Fuji' and 'Golden Delicious' reference genome. We selected mapping-validated 1,014 SNPs, most of which were heterozygous in 'Fuji' and capable of distinguishing alleles inherited from the parents of 'Fuji' (i.e., 'Ralls Janet' and 'Delicious'). We used these SNPs to define the haplotypes of 'Fuji' and trace their inheritance in relatives, which were shown to have an average of $27 \%$ of 'Fuji' genome. Analysis of variance (ANOVA) based on 'Fuji' haplotypes identified one quantitative trait loci (QTL) each for harvest time, acidity, degree of watercore, and mealiness. A haplotype from 'Delicious' chr14 was considered to dominantly cause watercore, and one from 'Ralls Janet' chr1 was related to low-mealiness.

Key Words: Malus $\times$ domestica, 'Fuji', SNPs, haplotype block, 'Ralls Janet', 'Delicious', watercore, mealiness.

\section{Introduction}

Apple (Malus $\times$ domestica Borkh.) is one of the most marketable and popular fruit crops; over $80 \mathrm{Mt}$ is produced per year throughout the temperate regions of the world (FAOSTAT 2013). In the Japanese fruit market, $0.8 \mathrm{Mt}$ of apples are produced per year, which is second only to orange (Ministry of Agriculture, Forestry and Fisheries of Japan [MAFF] 2014). Of domestic cultivated apple trees, $52 \%$ are 'Fuji', followed by 'Tsugaru' (13\%) and 'Orin' (8\%) (MAFF 2012). 'Fuji' was derived from the cross 'Ralls Janet' $\times$ 'Delicious' pollinated in 1939, and registered in 1962 (Sadamori et al. 1963). 'Fuji' has maintained its

Communicated by H. Iketani

Received February 3, 2016. Accepted April 19, 2016.

First Published Online in J-STAGE on July 8, 2016.

*Corresponding author (e-mail: miyuky@affrc.go.jp) overwhelming popularity for several decades, because of its juiciness, well-balanced taste, and long shelf-life. It can be stored until the beginning of the next summer, when it is adequately treated (i.e. cultivation of bagged-fruits or controlled atmosphere storage). Watercore frequently observed in the flesh of 'Fuji' is welcomed by Japanese consumers as an index of a well-ripened fruit. 'Fuji' is globally considered to be a major cultivar; it has the largest share of world production, with a greater than $45 \%$ share of production in China, the top producing country (O'Rourke 2003). However, 'Fuji' has some drawbacks, such as insufficient skin coloration or susceptibility to some diseases. Therefore, a new, attractive cultivar that is differentiated from 'Fuji', but holds the advantages of 'Fuji' is desired.

Breeders have frequently used 'Fuji' or its descendants as the parents of breeding populations, and a large number of cultivars or breeding lines derived from 'Fuji' are grown in Japan. As of 2015, the 199 apple cultivars registered with 
MAFF include 83 derived from 'Fuji'. Owing to the release of the whole genome sequence of 'Golden Delicious (GD)' (Velasco et al. 2010), it has become easy to re-sequence and assemble the genomes of apple varieties. Our objective here was to detect QTLs responsible for the superior characteristics of 'Fuji', by using sequence information of 'Fuji' and many of its relatives. QTLs for several traits have been previously reported on the genetic map of 'Fuji', or the integrated maps of the populations generated by crossing 'Fuji' with other cultivars; i.e., a QTL for firmness (Costa et al. 2010), QTLs for some other physical texture components (Longhi et al. 2012), and QTLs for some volatile organic compounds (Cappellin et al. 2015). Here, we focused on the degree of watercore and flesh mealiness as an index of fruit shelf-life. The development of mealiness during storage does not always correspond with the firmness or softening of fruit flesh; i.e., softening is often observed without mealiness in some cultivars (Iwanami et al. 2005).

The most popular and precise method for detecting QTLs in apple, e.g., interval mapping, is the linkage-based QTL analysis by using full-sib family and genetic map under a double pseudo-test cross strategy; this method was used in the QTL studies described above. Alternatively, a genomewide association study, which requires neither linkage map nor pedigree information, can be performed using breeding populations towards the application of genomic selection (Kumar et al. 2013). Recently, pedigree-based QTL analysis (PBA) has been applied to the detection of QTLs and the prediction of breeding value of seedlings (Bink et al. 2008, 2014). In genome-wide association or PBA studies, there is no need to maintain a large full-sib family, and a population consisting of visible genotypes or small families is sufficient. As the number of developed markers becomes huge, the "haplotype" definition of pedigree becomes popular for understanding allele transmission from parents to offspring (Bink et al. 2012, Voorrips et al. 2012). "Haplotype" is a combination of polymorphisms on a single chromatid detected by genetic markers linked on a chromosome. "Haplotype block" is a chromosomal segment represented as a cluster of tightly-linked markers. The "haplotypes" in a "haplotype block" are likely to be conserved among descendants through generations. In rice, the haplotypes of Japanese elite cultivar 'Koshihikari' and its relatives were defined by using genome-wide SNPs, which revealed the ancestral origins of the 'Koshihikari' genome and provided important information for rice breeding (Yamamoto et al. 2010). Here, we defined the haplotypes of 'Fuji' and its relatives, and conducted haplotype-based analysis, a simple kind of PBA combining the pedigree information and ANOVA, to develop markers effective for the selection of desirable phenotypes inherited from 'Fuji'.

We report the re-sequencing of 'Fuji', the detection of variants in the 'Fuji' genome (compared with the 'GD' genome), the development of SNP markers capable of distinguishing the two alleles of 'Fuji' at $1 \mathrm{cM}$ intervals, the identification of haplotypes of 'Fuji' and their inheritance through generations, and the detection of QTLs for the characteristic traits of 'Fuji' (e.g., degree of watercore and mealiness) based on haplotypic information.

\section{Materials and Methods}

\section{Plant materials}

Material for the re-sequencing of 'Fuji' was obtained from a tree grown in a field of the NARO Institute of Fruit Tree Science, Apple Research Station (Morioka, Japan). For the validation of designed SNP markers through genetic map construction, we used 96 seedlings in the juvenile phase generated from the cross 'Fuji' $\times$ 'GD'. In the haplotype analyses, 115 accessions related to 'Fuji', most of which were domestic ones, were used (Table 1). These accessions included the two parents of 'Fuji' (i.e., 'Ralls Janet' and 'Delicious'), 'Fuji' itself, and several generations derived from these founders. The accessions had been cultivated with some repetition for 5-30 years. Various grafted rootstocks were used: e.g., JM1, JM7, JM8, M.9A, M.9EMLA, M.26, and M.26EMLA. All segregating populations and evaluated accessions were cultivated at the NARO Institute of Fruit Tree Science, Apple Research Station.

\section{Re-sequencing of 'Fuji'}

The genomic DNA of 'Fuji' was extracted from leaves by using a Genomic-tip 20/G kit (Qiagen, Hilden, Germany). DNA was fragmented with a LE220 Focused-ultrasonicator (Covaris, Woburn, MA, USA). The size-selected DNA (average length, $300 \mathrm{bp}$ ) was purified with gel extraction and AMPure XP (Beckman Coulter, Brea, CA, USA). A standard short-read library was built by using a TruSeq DNA Sample Prep v2 Kit and TruSeq SBS v5 kit (Illumina, San Diego, CA, USA) for sequencing runs at $2 \times 76 \mathrm{bp}$ total. After sequencing, GAIIx real-time analysis (RTA) 1.19.38.0 and CASAVA 1.8.0 (Illumina) were used for base calling. Low-quality bases and adapter sequences were trimmed, and then reads shorter than 32 bp or unpaired reads were discarded by using in-house scripts and cutadapt (Martin 2011). The pre-processed reads were aligned to the 'GD' reference genome v1.0p (Velasco et al. 2010) by using BurrowsWheeler Alignment tool v0.6.1 (Li and Durbin 2009), and then read pairs with high mapping quality $(>Q 20)$ that were properly aligned were selected by samtools $0.1 .18(\mathrm{Li}$ 2011). Local realignment around indels was performed by using Genome Analysis Toolkit v1.4-6 (GATK) (DePristo et al. 2011). Finally, PCR duplicates were removed by using Picard v1.61 MarkDuplicates (http://sourceforge.net/projects/ picard/).

\section{Detection and selection of SNPS for GoldenGate assay}

Variants were detected through the comparison between the aligned reads of 'Fuji' and the 'GD' reference genome by using both "UnifiedGenotyper" in GATK and "mpileup" in samtools. Biallelic variants that were detected in common by both programs with high variant quality $(>20)$ were 


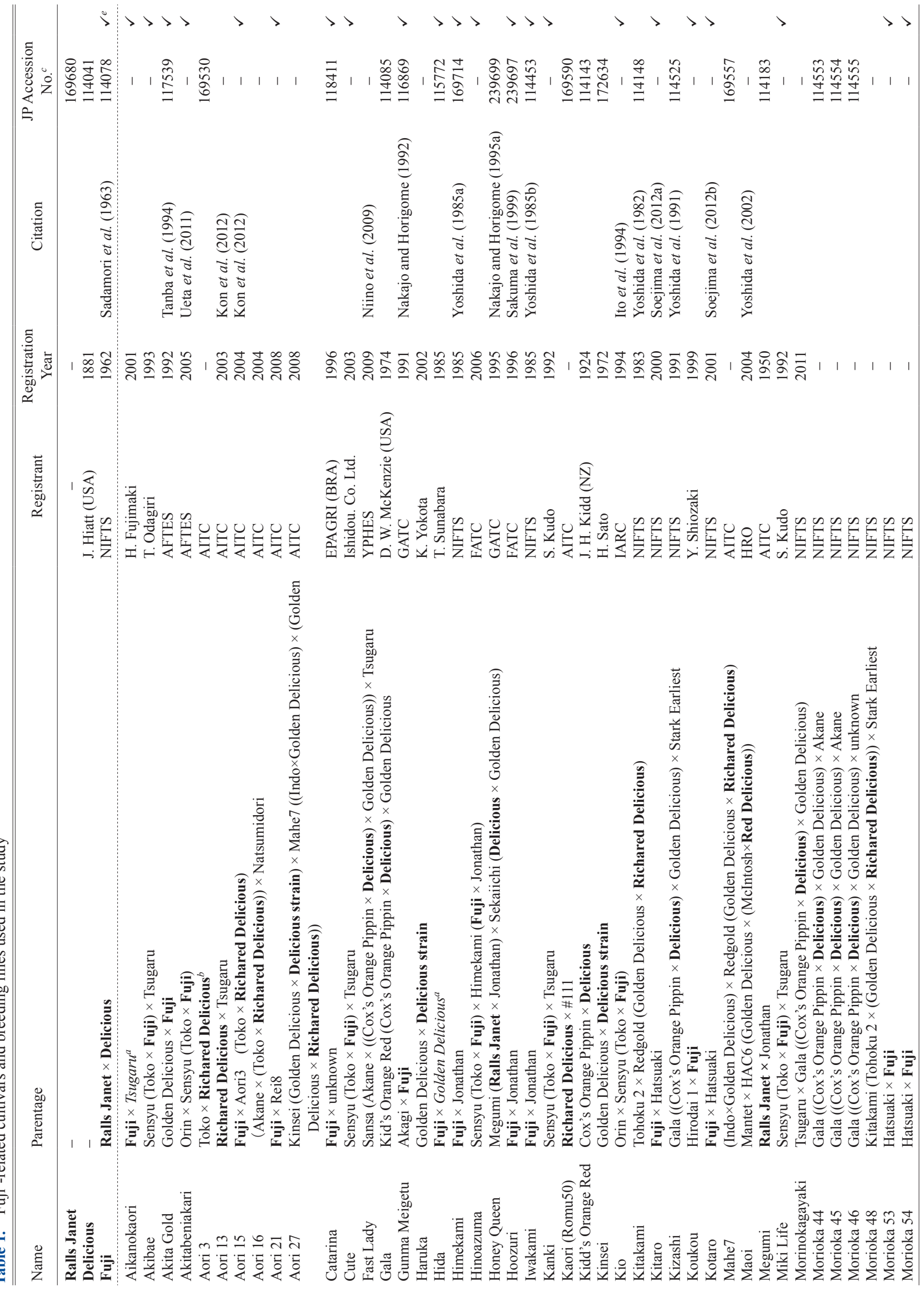




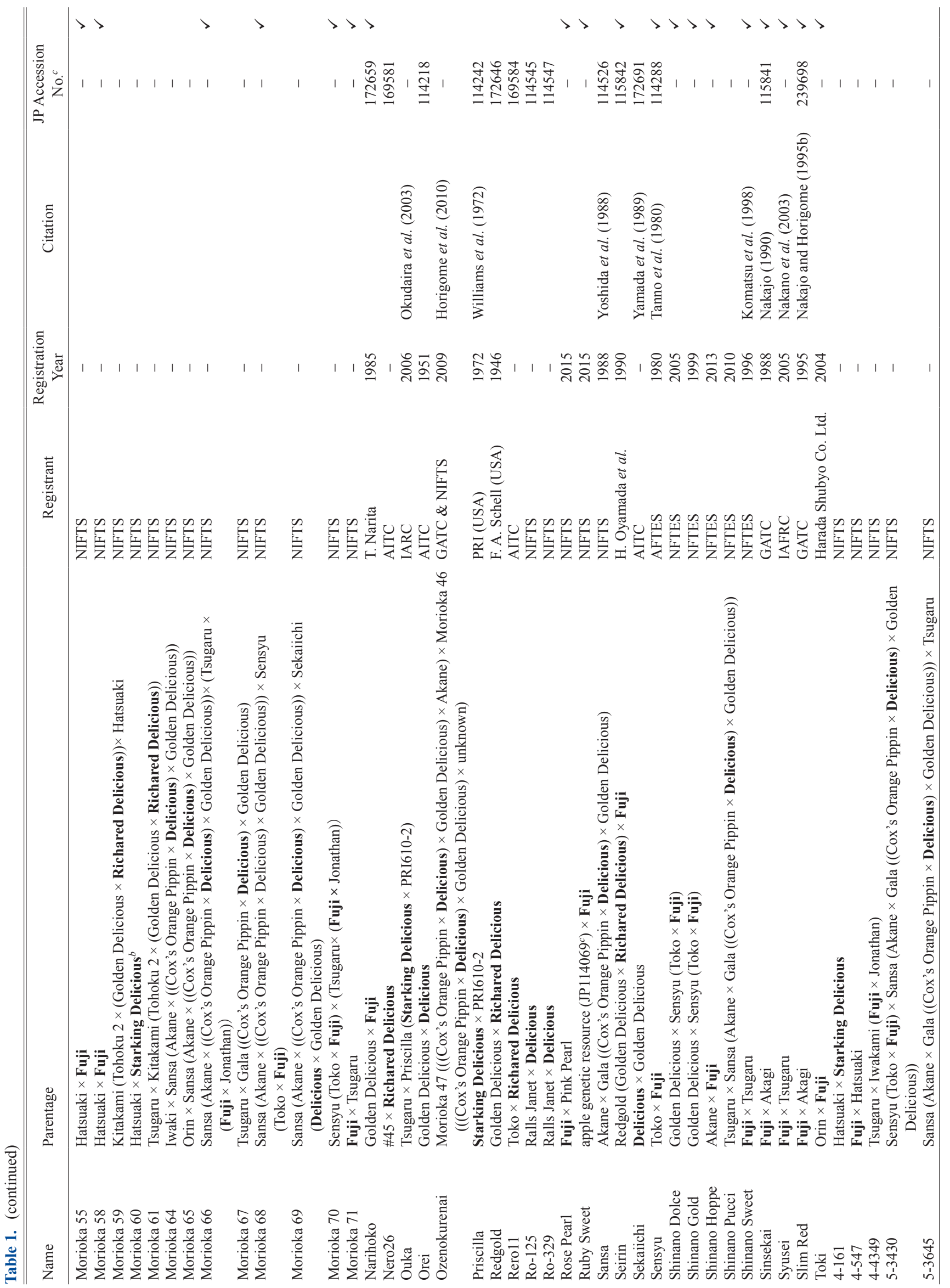


(I)

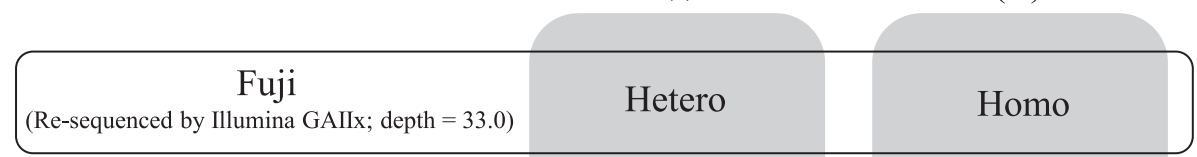

\begin{tabular}{|ccc|}
\hline $\begin{array}{c}\text { Golden Delicious } \\
\text { (Malus x domestica Whole Genome v1.0p) }\end{array}$ & Homo & Hetero \\
(Ambiguous nucleotide)
\end{tabular}

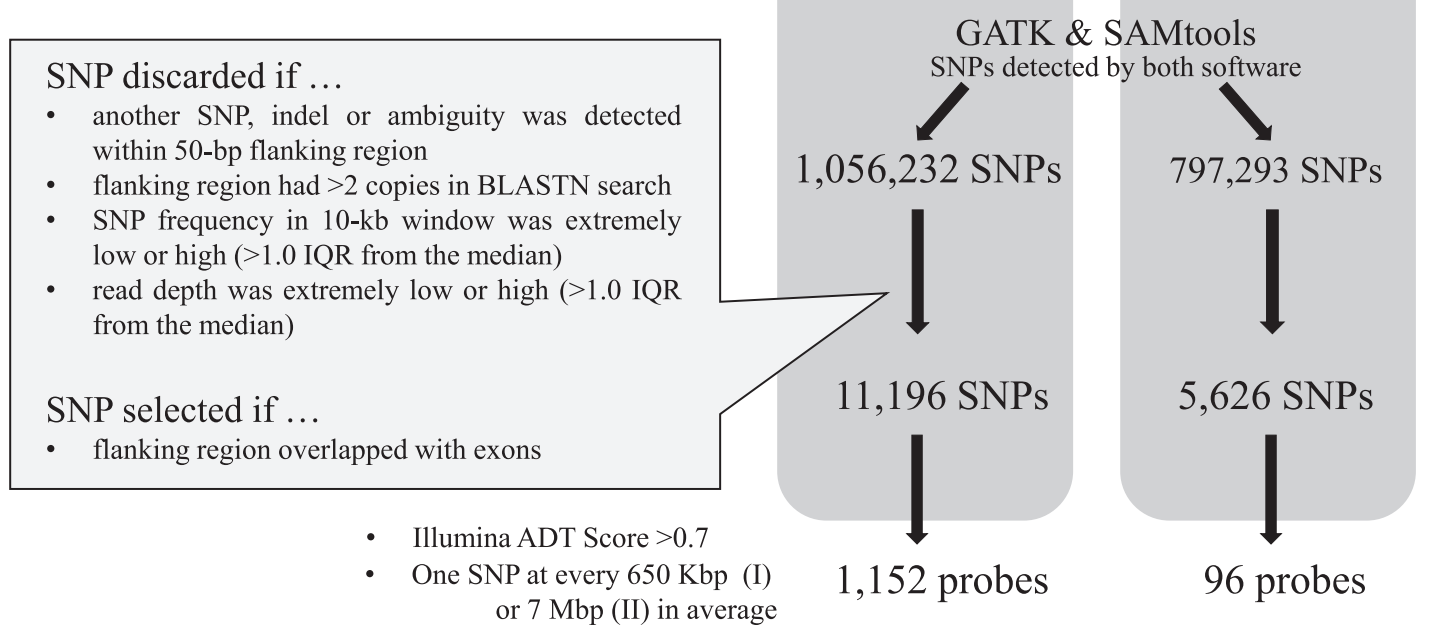

Fig. 1. Flow of SNP probe selection for GoldenGate Assay.

selected. Biallelic ambiguous sites (Y, K, R, M, W, and S) in the reference 'GD' genome were regarded to be heterozygous. Detected variants were divided into six categories: 1) SNPs that are homozygous in 'Fuji' and homozygous for another base in 'GD' (FJ/GD = hom/hom) (e.g., AA in 'Fuji' and GG in 'GD'); 2) SNPs that are heterozygous in 'Fuji' and homozygous in 'GD' ( $\mathrm{FJ} / \mathrm{GD}=$ het/hom) (e.g., $\mathrm{AG}$ in 'Fuji' and GG in 'GD'); 3) SNPs of FJ/GD = hom/het; 4) SNPs of $\mathrm{FJ} / \mathrm{GD}=$ het/het; 5) indels of $\mathrm{FJ} / \mathrm{GD}=$ hom $/ \mathrm{hom}$ (e.g., ++ in 'Fuji' and -- in 'GD'); and 6) indels of FJ/ $\mathrm{GD}=$ het $/$ hom (e.g., +- and -- ).

Among these variants, SNPs in category $2(\mathrm{FJ} / \mathrm{GD}=$ het/ hom) were selected as target sites for SNP genotyping by GoldenGate assay (Illumina), because they are most suitable for construction of 'Fuji' map using a 'Fuji' × 'GD' segregating population. Furthermore, the heterologous SNPs in 'Fuji' could be used as markers to trace the inheritance of each allele to its descendants. To select reliable marker SNPs, we discarded SNPs meeting one or more of the following criteria (Fig. 1): 1) other variants (i.e., SNPs, indels) or ambiguous bases in the 'GD' reference genome were detected within the 50-bp flanking regions on either side of the SNP; 2) 50-bp flanking sequences on either side of the SNP were aligned to two or more regions in the 'GD' reference genome by BLASTN search; 3) SNP frequency in the 10-kb window including the SNP was extremely low or high (i.e., $<8$ or $>34$; less than or greater than 1.0 interquartile range from the median); and 4) the depth of coverage of 'Fuji' reads was extremely low or high (i.e., $<12$ or $>56$; less than or greater than 1.0 IQR from the median). Lastly, we select- ed SNPs with the flanking 50-bp regions overlapped with annotated exons, in order to develop the markers extremely close to functional genes. The SNP probes designed for exonic regions were also expected to be stable in various accessions, because of lower levels of polymorphisms. Among selected SNPs, those that were unsuitable for probe design because they had a SNP score under 0.7 calculated by Illumina Assay Design Tool (ADT), or were an A/T or C/G transversion, were discarded. The final selected 1,152 SNPs were distributed evenly over the genome at an average interval of $0.65 \mathrm{Mbp}$; an even distribution was impossible to achieve in some regions because of lack of adequate SNPs.

Ninety-six SNPs of category $3(\mathrm{FJ} / \mathrm{GD}=$ hom/het) were also selected for array at approximately 7-Mbp intervals, following the filtering process described above. These markers were developed for the evaluation and comparison of accuracy of SNP prediction between 'Fuji' and 'GD' genome sequences.

\section{Validation of SNPs and genetic linkage maps}

We evaluated the availability and physical position of each selected SNP through the map construction. DNA was extracted from the segregating population and its parents ('Fuji' and 'GD') by using a DNeasy Plant Mini Kit (Qiagen), and genotyped by using GoldenGate assay following Illumina's instructions. Genotyping data were analyzed by using GenomeStudio software (Illumina) with a GenCall threshold of 0.25 . Clusters were manually edited so that parent-offspring segregation would be correct. SNPs with a GenTrain score under 0.6 or a cluster separation score under 
0.4 were visually checked and judged whether they should be discarded. Genotype calls of 'Fuji' and 'GD' were compared with those predicted by re-sequencing.

A genetic map was constructed by using all available SNP markers (FJ/GD = het/hom, hom/het, and accidentally obtained het/het), in addition to 475 simple sequence repeat (SSR) markers to ensure the accuracy and the direction of the linkage groups (LGs). The source of SSR markers and analytical protocol were same as those reported previously (Kunihisa et al. 2014). Construction of an integrated linkage map was carried out under a double pseudo-test-cross mapping strategy by using JoinMap 4.0 (van Ooijen 2006). Marker genotypes were converted to "CP (cross-pollinated)" type code and grouped under the following conditions: regression mapping module, LOD $=7.0$ and Kosambi's map function. We numbered the constructed LGs and validated the SSR order by referring to apple reference maps of 'Fiesta' and 'Discovery' (Liebhard et al. 2003a, SilfverbergDilworth et al. 2006).

The markers predicted to be located on LG3 and LG11 were simultaneously mapped on a single LG, and couldn't be separated into two LGs even by changing the grouping conditions. Among these markers, we selected those transformable to "BC1 (backcross population)" type code, and reconstructed LGs in 'Fuji' and 'GD', respectively, under the same conditions as the "CP" population mapping. The separated LG3 maps of 'Fuji' and 'GD' were integrated by using SSR loci as anchors. The same applied to LG11 maps.

\section{Definition of haplotypes of related accessions}

DNA was extracted from 115 accessions (Table 1) and genotyped with mapped SNPs by following the method used in the previous section. The SNPs were excluded from the haplotype analyses when the genotype clusters visualized by GenomeStudio were not clearly separated among 115 accessions. The SNPs genotype data were arranged in order according to the mapped position. The method used for identification of haplotypes in the 115 accessions is illustrated in Fig. 2. The first step was the phasing of the 'Fuji' genotype. For each chromosome, phases were judged from the linkage of genotypes among adjacent SNPs by using F1 progeny from the 'Fuji' $\times$ 'GD' cross. Majority linkage between polymorphisms was defined as coupling phase and minority as repulsion phase. The second step was the haplotype phasing of 'Ralls Janet' and 'Delicious' to identify haplotypes that were inherited by 'Fuji'. Haplotypes that were not inherited by 'Fuji' were kept undefined in this study. For the phasing of 'Ralls Janet', we examined the linkage among SNPs derived from 'Ralls Janet' by using four accessions (including 'Fuji') whose parent was 'Ralls Janet'. The phasing of 'Delicious' was carried out in a similar manner by using 10 accessions whose parent was
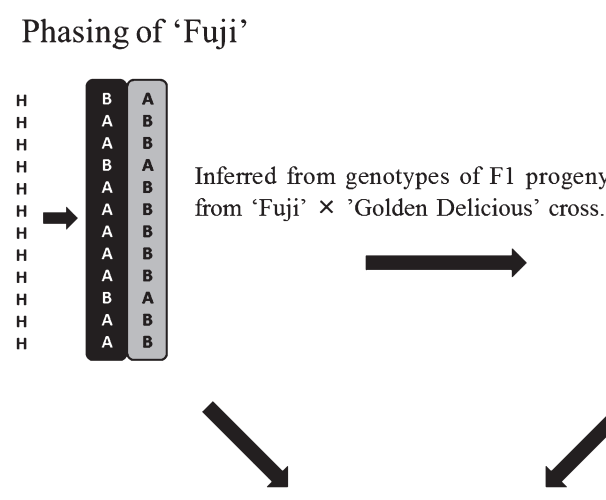

Search for haplotypes of 'Fuji' inherited by relatives

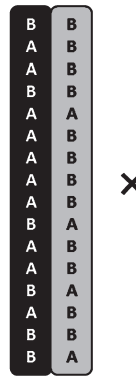

'Fuji'

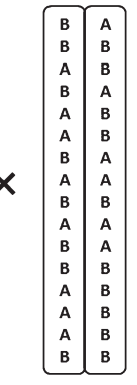

Another parent

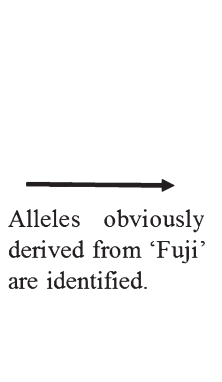

F1 cultivar

Phasing of parents of 'Fuji'
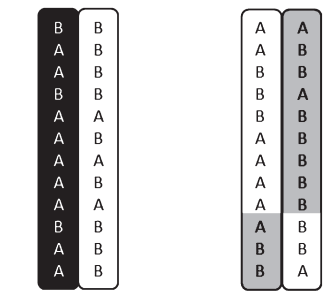

Inferred from genotypes of 4 or 10 cultivars, whose parent is 'Ralls Janet' or 'Delicious' strain, respectively.

\section{'Ralls Janet' 'Delicious'}

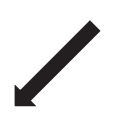

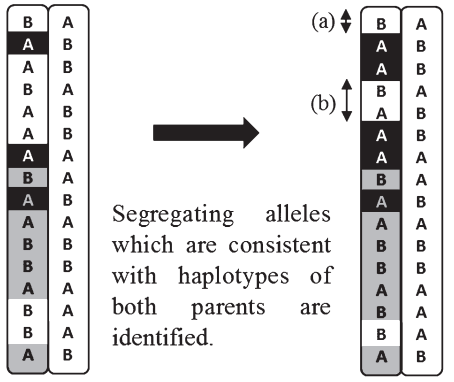

\begin{abstract}
The unidentified block is assumed to be included in the adjacent blocks, if it is ...

(a) $<5 \mathrm{cM}$, at the end of chromosome (recombination $P<0.05$ )

(b) $<20 \mathrm{cM}$, between the blocks from same origin (double recombination $P<0.04$ ) and consistent with haplotypes of
\end{abstract} both parents.
Complete

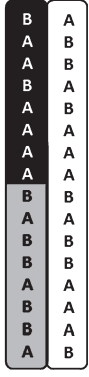

Fig. 2. Method for identification of haplotypes inherited by relatives of 'Fuji'. "H" represents a heterozygous genotype at a SNP position. Black and grey cells represent 'Fuji' alleles derived from 'Ralls Janet' and 'Delicious', respectively.

Some loci are inverted or discarded to decrease the discrepancies in the haplotype block. 
'Delicious'. The last step was to trace the propagation of the haplotypes of 'Fuji' in its relatives or descendants. Considering the relationship between two parents and their offspring, the alleles of SNPs obviously derived from target haplotypes were defined. Subsequently, those that segregated in concert with haplotypes of both parents were identified. When an undefined haplotype block was under $5 \mathrm{cM}$ from the end of a chromosome and was consistent with the haplotypes of both parents, it was assumed to be included in the adjacent block (because the risk of recombination was $<0.05$ ). Similarly, if an undefinable haplotype block was smaller than $20 \mathrm{cM}$ that was located between blocks from the same parental origin, it was assumed to be included in the adjacent blocks (because the risk of double recombination was $<0.04$ ). An undefinable block remained unidentified if it was placed between blocks of different origins. Finally, some SNP loci were manually rearranged or discarded to decrease the discrepancies in the haplotype blocks.

\section{Evaluation of apple traits}

Fruits from individual accessions were harvested when the ground color at the calyx end changed from green to yellowish-green. Our target traits were harvest time, fruit weight, acidity, degree of watercore and flesh mealiness. Four set of phenotypic data (harvest day, fruit weight, acidity, and degree of watercore) were obtained from 1991 to 2013 by using 108-115 accessions, respectively (Supplemental Table 1). The various accessions were evaluated for 1-23 years (average, 5.7 years). Harvest day was scored as the number of days after July 1st. On the day of harvest, by using 3-5 fruit of middle size, fruit weight and titratable acidity were measured, and the degree of watercore in the equatorial plane of the fruit was visually scored $(0$, nil; 1 , slight; 2 , moderate; 3 , strong), according to the guidelines for apple production (Aomori Prefectural Apple and Fruits Division et al. 2014). The phenotypic data were compensated based on inter-year variation, calculated by using the data of standard accessions that were thoroughly evaluated for 23 years. In the degree of watercore, some samples showed negative value because of the compensation, and these values were considered as 0 . The average of compensated data in multiple years was used as the representative value for the accession.

Flesh mealiness was measured in 2013 and 2014 by using the 63 accessions indicated in Supplemental Table 1, due to the difficulty of measurement of this trait. With the exception of some accessions, we used same trees in both years. Five fruit were measured per accession unless an individual did not have sufficient fruit, in which case all fruit were used in the analysis. The fruits were kept under $20^{\circ} \mathrm{C}$, $85 \% \pm 5 \%$ relative humidity for 4 weeks after the harvest and then evaluated by using the quantitative method reported by Iwanami et al. (2005). This method can measure the degree of cell separation by shaking flesh tissue discs in a sucrose solution; the degree of mealiness was calculated as (Wi-Ws)/Wi where Wi and Ws are weight of discs before and after the shake, respectively. Negative values were replaced with 0 . Negative values indicate an increase of fruit disc weight caused by the filling of the air space of discs with sucrose solution during shaking. The data were reported as the average of the two years.

\section{QTL detection using haplotype data}

We used the identified haplotype data of 108-115 accessions, described above, in the detection of QTLs for harvest time, fruit weight, acidity, degree of watercore, and those of 63 accessions, described above, for detection of QTLs for degree of mealiness. At each SNP locus, alleles identical to 'Fuji' were respectively coded as " 0 " or " 1 ", depending on whether the alleles were derived from 'Ralls Janet' or 'Delicious'. For each SNP, we tested the difference of phenotypic means between the accessions possessing the " 0 " allele and those possessing the "1" allele using t-test implemented in R program, where P-value $(P)$ was calculated for each SNP. In this statistical test for SNPs, the accessions possessing no alleles identical to 'Fuji' or those possessing both of "0" and " 1 " alleles were omitted from the analysis. The threshold of significance was set at $P=0.05 \times 10^{-3}$, because the tests were repeated at approximately 1,000 SNPs. This threshold value of $\mathrm{P}$, however, was likely to be conservative due to the inclusion of closely linked multiple SNPs on a haplotype block that could be regarded as a single SNP cluster. The calculated P-values were plotted against SNPs in the form of a Manhattan plot.

\section{Results}

\section{Re-sequencing of 'Fuji' and detected polymorphisms}

Sequencing of the 'Fuji' genome resulted in 294 million read pairs (44.6 Gb in total; DRA004333, registered in the DDBJ Sequence Read Archive). After pre-processing, 244 million read pairs were retained. Of these, 84 million read pairs (corresponding to $12.7 \mathrm{~Gb}$ ) were finally mapped to the 'GD' reference genome, with an average of $33 \times$ depth of coverage. The total length of genomic regions covered with at least one read was $346 \mathrm{Mbp}$, which corresponds to $57 \%$ of the total 'GD' reference genome, and $90 \%$ of the reference genome without Ns or ambiguous bases. The obtained sequences of 'Fuji' were positioned across all 17 chromosomes, and were sufficient for marker development.

Two kinds of software called a total of 2,820,759 variants including SNPs and indels in common between the genome sequences of 'Fuji' and 'GD'. Among the SNPs, 551,506 were found to be $\mathrm{FJ} / \mathrm{GD}=\mathrm{hom} / \mathrm{hom}, 1,056,232$ were $\mathrm{FJ} / \mathrm{GD}=$ het/hom, 797,293 were $\mathrm{FJ} / \mathrm{GD}=$ hom/het, and 251,297 were $\mathrm{FJ} / \mathrm{GD}=$ het/het. Among the indels, 97,757 were $\mathrm{FJ} / \mathrm{GD}=\mathrm{hom} / \mathrm{hom}$ and 66,674 were $\mathrm{FJ} /$ $\mathrm{GD}=$ het $/$ hom

\section{Selection of SNPS}

The detected 1,056,232 SNP variants of FJ/GD = het/ hom were filtered according to the annealing characteristic 
Table 2. Result of GoldenGate assay of designed SNP probes and genetic mapping

\begin{tabular}{|c|c|c|c|c|c|c|c|c|c|}
\hline \multirow{2}{*}{$\begin{array}{l}\text { Predicted } \\
\text { zygosity for } \\
\text { FJ/GD }\end{array}$} & \multirow{2}{*}{$\begin{array}{l}\text { \# of designed } \\
\text { SNP probes }\end{array}$} & \multirow{2}{*}{$\begin{array}{c}\text { \# of genotyped } \\
\text { SNPs }\end{array}$} & \multicolumn{4}{|c|}{ Observed zygosity for FJ/GD } & \multirow{2}{*}{$\begin{array}{l}\text { \# of mapped } \\
\text { SNPs }\end{array}$} & \multirow{2}{*}{$\begin{array}{l}\text { \# of mapped } \\
\text { SNPs on the } \\
\text { predicted loci }\end{array}$} & \multirow{2}{*}{$\begin{array}{c}\text { \# of SNPs used } \\
\text { for haplotype } \\
\text { definition }\end{array}$} \\
\hline & & & het/hom & het/het & hom/het & hom/hom & & & \\
\hline het/hom & 1152 & 988 & 830 & 148 & 3 & 7 & 980 & 765 & 938 \\
\hline hom/het & 96 & 90 & 0 & 0 & 78 & 12 & 77 & 73 & 76 \\
\hline
\end{tabular}

$\mathrm{FJ} / \mathrm{GD}=$ het/hom means that the genotype of each SNP is heterozygous in 'Fuji', and homozygous in 'Golden Delicious'. The same applies to $\mathrm{FJ} / \mathrm{GD}=$ hom/het, het/het and hom/hom.

and uniqueness of flanking sequences as the SNP probe (see "Materials and Methods", Fig. 1); this process removed 91.2\% of SNPs, leaving 92,766 SNPs. After applying further filters for adequate SNP frequency and read depth, and exonic location, 11,196 SNPs were retained. Of these, we selected 1,152 SNPs (MdFJ set), after considering the ADT score of the SNP probes and the intervals between them (Supplemental Table 2), for the GoldenGate assay.

We also discarded $99.3 \%$ of the called 797,293 SNPs of $\mathrm{FJ} / \mathrm{GD}=$ hom/het by using the filters described above, to obtain 5,626 exonic reliable SNPs. On the basis of the ADT score and intervals between SNPs, we further selected 96 SNPs (MdGD set) for the GoldenGate assay.

\section{Validation of selected SNPs and mapping}

The segregating population from the 'Fuji' $\times$ 'GD' cross were genotyped by GoldenGate assay or SSR marker set, and an integrated linkage map was generated. Of the 1,248 SNP probes (i.e., MdFJ and MdGD sets combined), 1,078 showed informative segregation in the population, and 1,057 were successfully mapped with 475 SSR markers spanning 1,130.6 cM on 17 chromosomes (Table 2, Supplemental Fig. 1). The order of inserted SSR markers was mostly consistent with previous reports (SilfverbergDilworth et al. 2006, Kunihisa et al. 2014). The average interval between SNP markers was $1.07 \mathrm{cM}$. Of the 1,057 mapped SNPs, 838 were mapped on the positions consistent with the prediction on the 'GD' reference genome, and 219 were on conflicting positions. Although as many as $1 / 5$ of SNPs were mapped on unexpected positions, each linkage group was covered with a sufficient number of SNPs. Among the mapped SNPs, 43 were removed in the haplotype analyses, because of unclear genotype separations among the accessions from various pedigrees, or because of an obvious discrepancy in the haplotype block. Finally, a total of 1,014 SNPs were used for the following study.

The genotypes of 'Fuji' and 'GD' determined by GoldenGate assay were compared to those predicted through re-sequencing. Eighty-four percent (830 out of 988) of SNPs predicted as FJ/GD $=$ het/hom and $87 \%$ (78 out of 90$)$ of those predicted as $\mathrm{FJ} / \mathrm{GD}=$ hom/het were detected as the corresponding genotypes (Table 2 ).

\section{Propagation of haplotypes common to 'Fuji'}

We traced the haplotypes derived from 'Fuji' as they were propagated in its relatives (Supplemental Table 3).
Of the SNP alleles in 115 accessions, an average of $27 \%$ were defined to be in common with either of heterologous alleles of 'Fuji', with an average of $11.5 \%$ and $15.5 \%$ being alleles derived from 'Ralls Janet' and 'Delicious', respectively. The undefined SNP alleles (73\%) were either derived from other origins, or unable to be judged their origin due to homozygous SNPs. The six chromosomes of 'Ralls Janet' (chr3, chr13-chr17) and 13 chromosomes of 'Delicious' (chr1, chr3-chr5, chr7-chr9, chr11, chr12, chr14-chr17) were recombined and inherited by 'Fuji'.

The 115 relatives of 'Fuji' could be divided into 1) the descendants of 'Fuji'; and 2) the full-sib or half-sib families of 'Fuji' and their descendants (Fig. 3). In the first-degree descendants of 'Fuji' (e.g., 'Sensyu'), almost all of the haplotypes inherited from 'Fuji' were identified as 'Fuji'; i.e, almost $50 \%$ of SNP alleles were defined to be in common with those of 'Fuji'. 'Aori15', 'Koukou' and 'Seirin' were the descendants that had more than $50 \%$ of SNP alleles in common with those of 'Fuji'; this was as expected because these are the results of crosses between 'Fuji' and a sport of 'Delicious' or its offspring. In the second-degree descendants of 'Fuji' (e.g., 'Shinano Gold'), most of which are offspring of 'Sensyu', fewer haplotypes of 'Fuji' could be observed, because of the weaker relationship to 'Fuji' and the increase in undefinable regions due to repeated recombination events. An exception was 'Hinoazuma', which showed as much as $48 \%$ of SNP alleles in common with those of 'Fuji'; this high percentage was as expected because 'Hinoazuma' is derived from a cross between two firstdegree descendants of 'Fuji'. For the same reasons as stated above, the sib families, most of which are descendants of 'Delicious', possessed smaller percentages of haplotypes in common with those of 'Fuji'. Basically, less haplotypes could be defined as the generations progressed.

The propagation of 'Delicious'-derived and 'Ralls Janet'-derived haplotypes of 'Fuji' in the 115 accessions deviated significantly from 1:1 at regions on chr1, chr7-chr9, and $\operatorname{chr} 15\left(P<0.05 \times 10^{-3}\right.$; Supplemental Fig. 2$)$ : the frequency of SNP alleles from 'Delicious' was higher than that of those from 'Ralls Janet' at regions on chr7-chr9, and chr15, and the reverse was observed for a region on chr1. The dominance of alleles from 'Delicious' was largely due to the more frequent use of 'Delicious' than 'Ralls Janet' as a cross parent. When we focused on 42 descendants of 'Fuji' selected as cultivars or superior breeding lines (shown with ticks in Table 1), a significant deviation was observed 


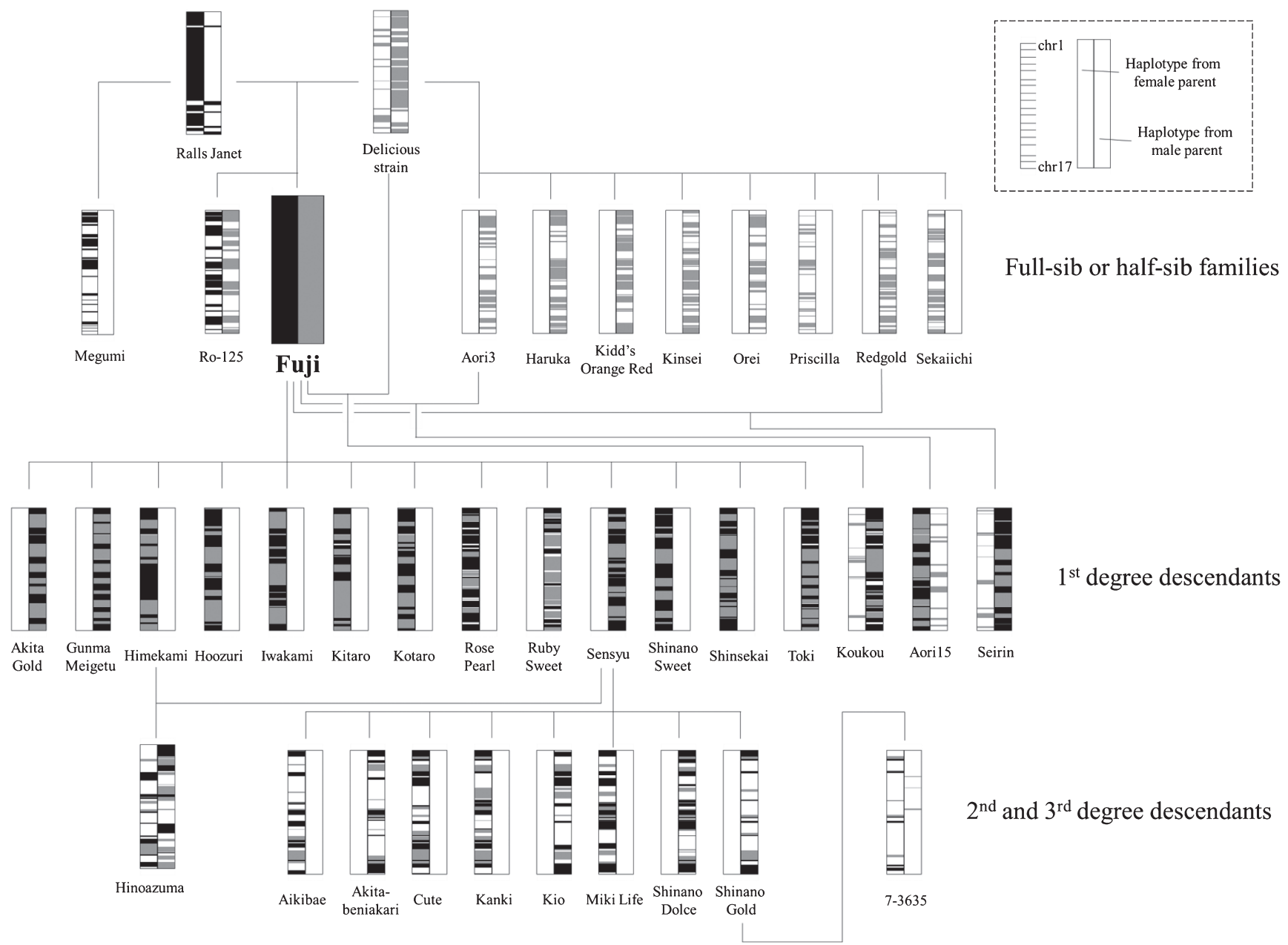

Fig. 3. The typical pedigree tree of 'Fuji' and the inheritance of 'Fuji' haplotypes. Black and grey blocks represent 'Fuji' haplotypes derived from 'Ralls Janet' and 'Delicious', respectively. White blocks include the following haplotypes: 1) those derived from founders other than 'Fuji'; 2) those that could not be judged to be derived from 'Fuji' or not; 3 ) those derived from 'Fuji', but which could not be identified its origin ('Ralls Janet' or 'Delicious').

only at a region on $\operatorname{chr} 1\left(P<0.05 \times 10^{-3}\right.$; Fig. 4). As much as $45 \%$ of haplotypes in this region were derived from 'Ralls Janet': i.e., $90 \%$ of descendant cultivars inherited the haplotype of 'Ralls Janet' through the generations. This result indicates that the genes in this region might have been under selection for the some kind of trait that was always targeted in crosses using 'Fuji' or 'Sensyu'.

\section{QTLs detected by ANOVA based on 'Fuji' haplotype}

The five phenotypes of the evaluated accessions are presented in Supplemental Table 1, and the distributions of them are shown in Supplemental Fig. 3. At each SNP locus, we tested phenotypic means of the accessions with the 'Ralls Janet'-derived alleles and those of the accessions with the 'Delicious'-derived allele by t-test, to detect QTLs for the five traits (Fig. 5, Table 3). A significant QTL $\left(P<0.05 \times 10^{-3}\right)$ for harvest day was found in the middle of chr16; one for acidity was detected in the upper middle of chr8; one for degree of watercore was detected in the middle of chr14; and one for degree of mealiness was located in the upper middle of chr1; no significant QTL was detected for fruit weight.

For the newly detected QTLs for degree of watercore and mealiness, the distributions of phenotypes among tested accessions with each haplotype are shown, in order to assess the effect of each haplotype (Fig. 6). A high degree of watercore (median, 1.05) occurred in accessions that possessed the haplotype from 'Delicious' around the MdFJ_1.036 marker; the degree of watercore was extremely low in accessions with the haplotype from 'Ralls Janet' (median, 0.09 ), or in those with other or undefined haplotypes (median, 0.08) in this region. The degree of mealiness was much higher (mean, 0.54) in accessions with the haplotype from 'Delicious' at the region between MdFJ_1.010-011 and MdFJ_2.017-019 than in those with the haplotype from 'Ralls Janet' (mean, 0.13). 

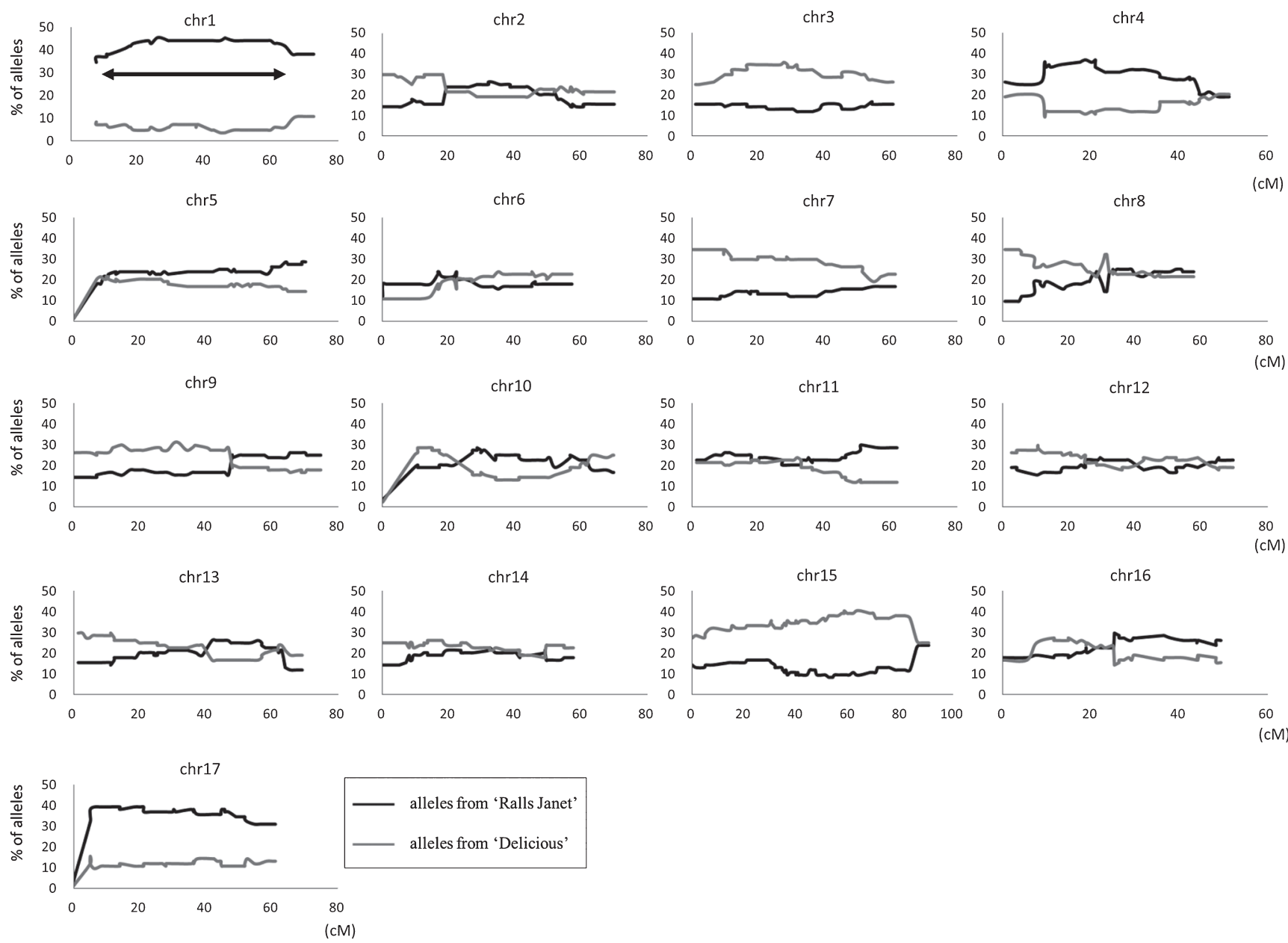

Fig. 4. Frequency of alleles inherited from 'Fuji' for 17 chromosomes in 42 descendant cultivars of 'Fuji'. Among the 115 accessions in Table 1, 42 descendants of 'Fuji' were selected as cultivars or superior breeding lines for further analysis. The alleles derived from 'Ralls Janet' and 'Delicious' were separately calculated. The region indicated by a two-way arrow showed frequency deviation from 1:1 between 'Ralls Janet' and 'Delicious' alleles at the level of $P<0.05 \times 10^{-3}$.

\section{Discussion}

\section{Accuracy and availability of designed SNP probes}

By re-sequencing the genome of 'Fuji' and comparing it to the 'GD' reference genome, we obtained 2.7 million SNP variants. During the development of the $20 \mathrm{~K}$ SNP Infinium array for apples (Bianco et al. 2014), 16.6 million SNPs among 14 re-sequenced accessions were scored. Although the software and the criteria for calls used in our study were different to those used in the previous study, we still detected about $1 / 6$ the number of variants with $1 / 8$ the number of reference accessions. Of the $11,196(\mathrm{FJ} / \mathrm{GD}=$ het/hom $)$ and $5,626(\mathrm{FJ} / \mathrm{GD}=$ hom/het $)$ SNPs exceeding the filtering criteria in this study, 212 and 57 were identical to previously developed SNP markers on $8 \mathrm{~K}$ or $20 \mathrm{~K}$ Infinium arrays (Bianco et al. 2014, Chagné et al. 2012). On the other hand, none of the 1,248 SNP markers designed for GoldenGate assays were identical to them. We also evaluated the accuracy of the genotypes predicted from the 'GD' reference genome and our re-sequencing of 'Fuji' genome (Table 2) by comparing the predicted genotypes to those obtained with GoldenGate assays. The accuracies of prediction for heterozygosity and homozygosity of 'Fuji' genotypes were $99.0 \%$ (978 out of 988) and $100 \%$ (90 out of 90), respectively. Those of 'GD' were $86.6 \%$ (78 out of 90 ) and $84.7 \%$ (837 out of 988). The higher prediction accuracy for genotypes of 'Fuji' compared with those of 'GD' is likely due to the very strict criteria we used in the read alignment, variant calling, and filtering steps to obtain reliable SNPs.

Of 1,248 designed SNP probes, 1,057 (84.7\%) were mapped in the 'Fuji' $\times$ 'GD' segregating population, and $1,014(81.3 \%)$ were used for the haplotype definition of various accessions. Some of these mapped SNPs (20.7\%) were located at positions that conflicted with predicted positions on the 'GD' reference genome. Such inconsistency was also observed in the map for M432 progeny constructed by Antanaviciute et al. (2012), using the $8 \mathrm{~K} \mathrm{SNP}$ infinium array, and is considered to be caused by unexpected paralogous genomic regions, or the mis-assignment of sequence contigs during the assembly. 
Harvest day

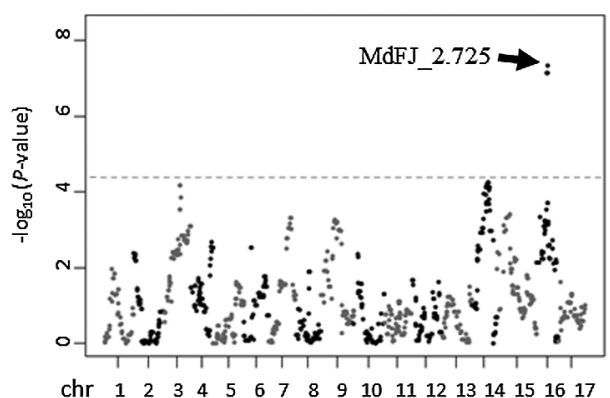

Acidity

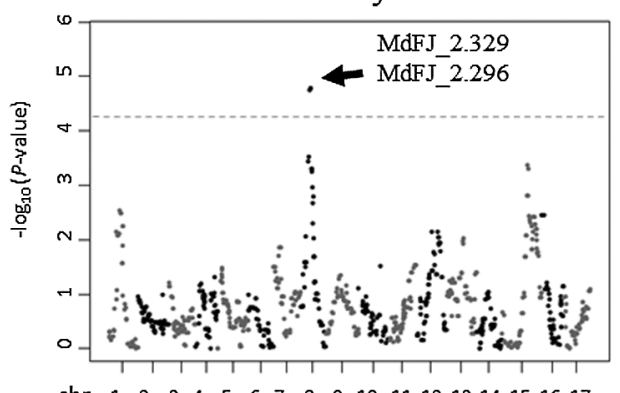

chr $1 \begin{array}{lllllllllllllllllll} & 2 & 4 & 5 & 6 & 7 & 8 & 9 & 10 & 11 & 12 & 13 & 14 & 15 & 16 & 17\end{array}$
Fruit weight

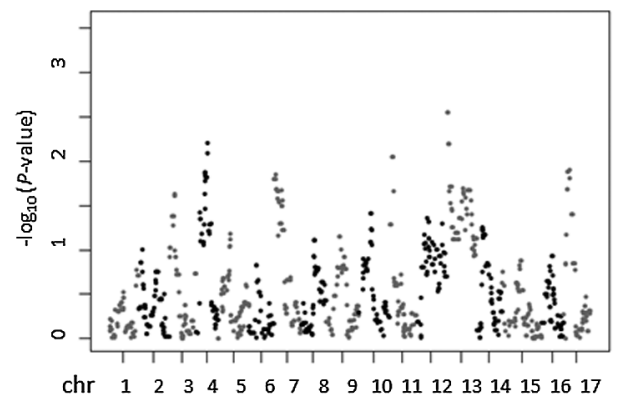

Degree of watercore

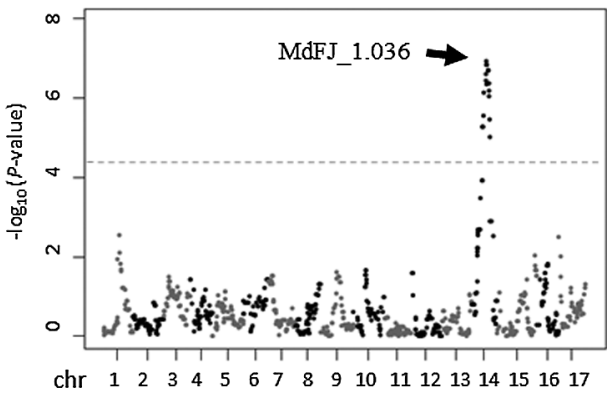

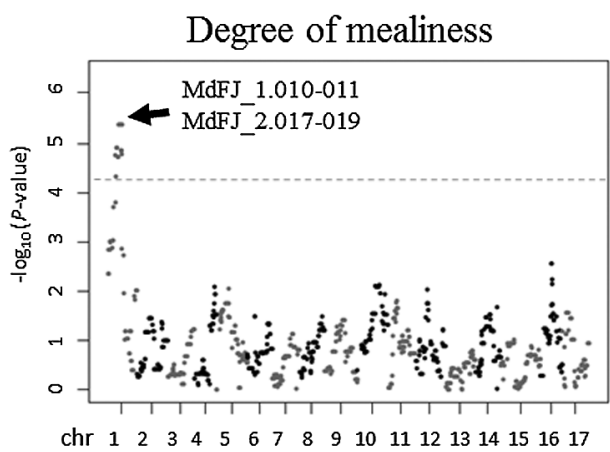

Fig. 5. QTLs detected by ANOVA using the haplotypes of 'Fuji'. For each SNP marker, we compared accessions with the allele from 'Ralls Janet' to those with the allele from 'Delicious'. Gray and black dots alternately represent data for individual markers on each chromosome, in order of Supplemental Table 3. Horizontal dashed line is the threshold set at $P=0.05 \times 10^{-3}$.

Table 3. Information regarding QTLs detected by comparing haplotypes of 'Fuji'

\begin{tabular}{lcccc}
\hline \hline Traits & LG & $\begin{array}{c}\text { Probability } \\
\left(-\log _{10}\right)\end{array}$ & $\begin{array}{c}\text { Significant } \\
\text { marker }^{a}\end{array}$ & $\begin{array}{c}\text { Position } \\
(\mathrm{cM})\end{array}$ \\
\hline Harvest day & 16 & 7.3 & MdFJ_2.725 & 24.9 \\
\hline Acidity & 8 & 4.8 & $\begin{array}{l}\text { MdFJ_2.329 } \\
\text { MdFJ_2.296 }\end{array}$ & $23.3-23.7$ \\
\hline Degree of watercore & 14 & 6.9 & MdFJ_1.036 & 32.4 \\
\hline Degree of mealiness & 1 & 5.4 & $\begin{array}{l}\text { MdFJ_1.010-011 } \\
\text { MdFJ_2.017-019 }\end{array}$ & $30.4-38.2$
\end{tabular}

LG, linkage group. The markers with minimum probability were listed with their position and $\mathrm{P}$-value.

${ }^{a}$ Significant means $P<0.05 \times 10^{-3}$.

\section{Propagation of haplotypes of 'Fuji'}

'Fuji' and its descendants have been especially frequently crossed to generate breeding populations, due to their high potential for breeding values; over $40 \%$ of cultivars registered with MAFF are derived from 'Fuji'. In Japanese rice breeding, 'Koshihikari' is the most-grown elite cultivar and has been repeatedly used as a cross parent. Yamamoto et al. (2010) defined the haplotype of 'Koshihikari' and related cultivars by using approximately 2,000 genome-wide SNP markers, and clarified that the $60 \%-80 \%$ of the genome of the second, third, and fourth most-grown cultivars is identical to that of 'Koshihikari', and that genetic variation has decreased in modern cultivars because of strong selection pressure. Inbreeding depression caused by repeated crossing within a narrow gene pool and genome homogeneity is becoming a serious concern in fruit tree breeding. In Japanese pear, there is a clear negative correlation between inbreeding coefficient and tree vigor, and the coefficient has been increasing in recent cultivars (Onoue et al. 2015). Therefore, we examined whether homozygosity of 

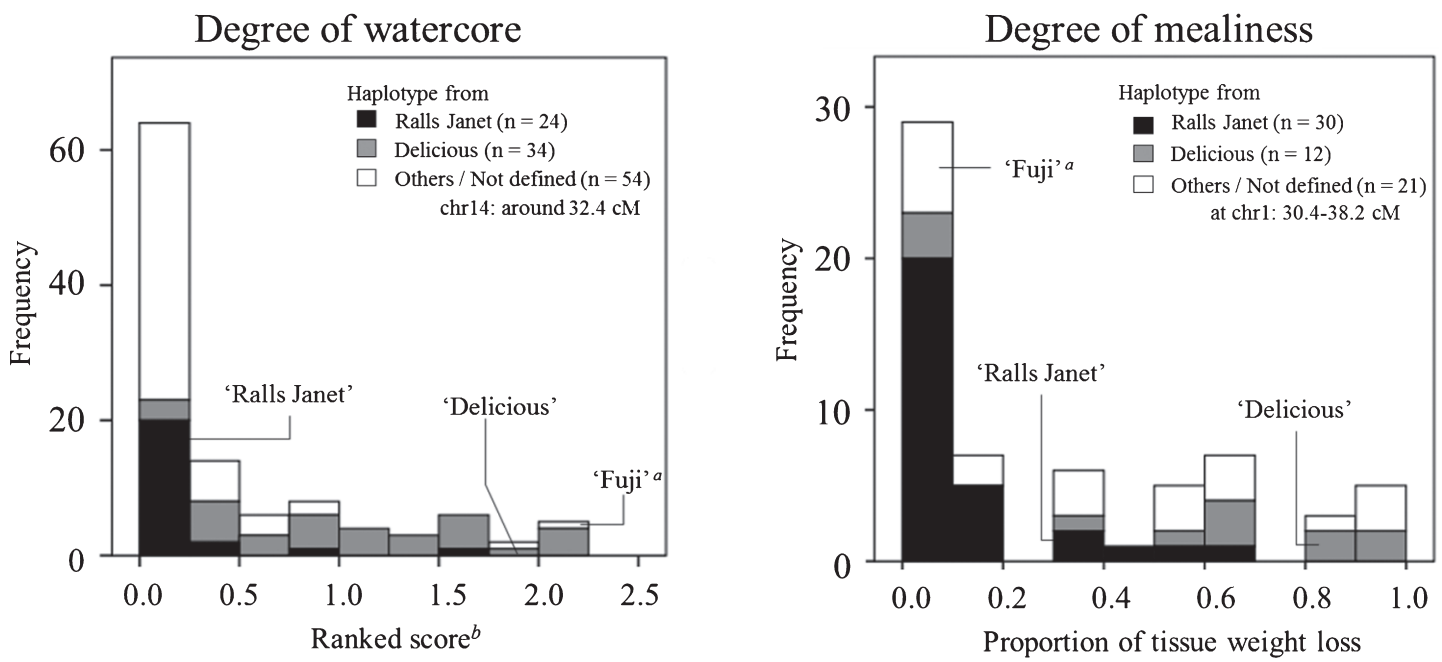

Fig. 6. Distribution of two phenotypes (degree of watercore and degree of mealiness) in tested accessions, depending on the haplotype at detected QTL. "Fuji' is classified as "Not defined", because it possesses haplotypes from both 'Delicious' and 'Ralls Janet' at the detected QTL. ${ }^{b}$ Ranked score (visual): 0 (nil), 1 (slight), 2 (moderate), 3 (strong).

haplotypes derived from 'Fuji' was present in recent accessions (Supplemental Table 3). Most of the accessions were free from inbreeding, at least for the haplotypes of 'Fuji', probably because the number of generations since the founders is less for Japanese apple breeding than for Japanese pear breeding. 'Aori 15', 'Hinoazuma', 'Koukou', and 'Seirin' showed relatively high homozygosity in several regions of chromosomes; the total length of them was $138 \mathrm{cM}, 76 \mathrm{cM}, 68 \mathrm{cM}$, and $59 \mathrm{cM}$, respectively.

However, a decrease in genetic variation might arise in the near future, because an extreme deviation between the frequencies of the 'Delicious'-derived and 'Ralls Janet'derived haplotypes of 'Fuji' was observed on chr1, at least, in descendant cultivars of 'Fuji' (Fig. 4). This deviation could have been caused either by the selection toward genes for desirable traits, or by the existence of bottleneck cultivars, such as 'Sensyu', with high-potential as a breeding parent.

\section{QTLs detected by haplotype-based analysis}

We detected one QTL each for harvest day, acidity, degree of watercore and mealiness. The QTL for harvest day, located on chr16, corresponded to one reported previously (Kenis et al. 2008, Kunihisa et al. 2014); likewise, the QTL for acidity, located on chr8, was identical to a known QTL (Kenis et al. 2008, Kumar et al. 2013, Liebhard et al. 2003b).

To our knowledge, this is the first report of QTL analysis and the development of linkage markers for degree of watercore. A high degree of watercore occurred in almost all accessions that possessed the 'Delicious' -derived haplotype of 'Fuji' in the middle of chr14 (Fig. 6); although three accessions with the 'Delicious' haplotype in this region had a degree of watercore score of 0 , watercore could be observed in two of them when the timing of harvest was later. In contrast, accessions with the 'Ralls Janet'-derived haplotype and a haplotype from other or undefined founders in this region rarely generated watercore. The only accession whose score was over 2.0 with an undefined haplotype was 'Fuji', which is coded "ND" because it possesses both haplotypes. These results indicate that the haplotype of 'Delicious' in this region dominantly causes watercore. The low degree of watercore in the accessions with a haplotype from other or undefined founders implies that other haplotypes (e.g., from 'GD' or 'Jonathan') hardly cause watercore.

Watercore development has been considered to correlate with several factors: high temperature, low calcium, maturation, and the decreased capacity of the cells to take up sorbitol (Marlow and Loescher 1984). In a study of 'Fuji', higher degree of watercore was associated with higher internal ethylene, sorbitol, or sucrose concentrations (Bowen et al. 1997). Gao et al. (2005) found that expression of the sorbitol transporter gene (MdSOT) was suppressed specifically in fruits with watercore, and that the defect of the membrane transport system of sorbitol caused the excessive sugar accumulation and watercore. However, a BLASTN search against the 'GD' reference genome showed that MdSOT genes were on chr3, chr12, or chr17, but not on chr14 where we identified a QTL.

Watercore of fruit tends to be recognized as an undesirable physiological disorder in many regions of the world, because it can cause browning or early breakdown of fruit flesh during storage. In contrast, it is greatly welcomed by consumers in Japan, where it is recognized as an index of a well-ripened fruit, and apples with watercore are dealt with at a higher price in the market. Therefore, the QTL detected in this study is available not only for negative but also for positive selection.

A QTL for degree of mealiness was detected at the middle of chr1; accessions having the haplotype of 'Ralls Janet' showed significantly lower mealiness (and therefore longer 
shelf-life). Most descendants of 'Fuji' inherited the haplotype of 'Ralls Janet' at the middle region on chr1 (Fig. 4), which could be the result of the selection for some important gene. From our results, we hypothesize that the breeders had focused on shelf-life in the screening of populations crossed with 'Fuji' or its descendants, and that selection for long shelf-life lines resulted in the discard of the haplotype of 'Delicious' that causes mealiness among cultivars at this QTL. We note that the accessions that inherited the haplotype of 'Ralls Janet' at this region largely overlapped with the descendants of 'Fuji', and those with the haplotype of 'Delicious' overlapped with other relatives. Other unevaluated traits could be candidates for the role of this biased region, if they are common only among the descendants.

Mealiness of apple flesh depends on low adhesion between neighboring cells, and high resistance to cell rupture (Harker and Hallett 1992). Nobile et al. (2011) reported that the gene expression and enzyme activity of $\alpha$-L-arabinofuranosidase $(\alpha$-AFase), acting on the pectic component of the cell wall, were correlated with mealiness. By transcriptome analysis, the expression of a pectin methylesterase gene (MdPME2) was found to be significantly higher in non-mealy fruits than in mealy fruits during fruit development, ripening, and post-harvest (Segonne et al. 2014). However, $\alpha$-AFase and MdPME2 are located on chr16 and chr9, respectively, not on chr1. Probes for 15 SNP markers with significant P-value were mapped to the region between chr1: $9.7 \mathrm{Mb}-16.1 \mathrm{Mb}$ of the 'GD' reference genome v1.0. The $M d-\operatorname{Exp} 7$ gene, which was deemed to be associated with fruit firmness or softening in analyses using progeny of 'Prima' or cultivar allele mining (Costa et al. 2008), was mapped to quite near this region $(16.6 \mathrm{Mb})$. The product of $M d$-Exp7 promotes cell wall relaxation and extension; therefore, it is a strong candidate gene not only for fruit firmness or softening, but also for mealiness. Although the SNP marker nearest to $\mathrm{Md}$ $\operatorname{Exp} 7$ (MdFJ 2.023, 16.6 Mb, $42.7 \mathrm{cM}$ ) was not significant, the extremely low-frequency of 'Delicious'-derived haplotype around this region (see Supplemental Fig. 2) might have hampered the adequate test of difference. Costa et al. (2010) carried out QTL analysis for fruit firmness or softening by using a full-sib population of 'Fuji', but detected no QTLs on chr1, where we detected a QTL for mealiness based on haplotypes of 'Fuji'. This might indicate that the causative gene for fruit firmness or softening and the gene for mealiness are not identical, or that the effects of the causative gene (probably $M d-\operatorname{Exp} 7$ ) for these traits were not in phase among accessions. The complex relationships among these traits for fruit texture are consistent with the report by Iwamani et al. (2005), which proposed that these traits were not in concert with each other, based on the existence of cultivars softened without mealiness. Further genetic and physiological analyses are required to clarify the relationships among these traits.

One of the advantages of haplotype-based analysis is the identification of the haplotypes that leads to a desirable phenotype; polymorphisms of a single marker cannot always distinguish between the exact allele with the desirable effect and other alleles. The ANOVA between the haplotypes of 'Fuji' used in this study could be substituted for the conventional method using full-sib family and pseudo-testcross mapping strategy, and could compensate for the lack of fullsib family in the QTL study.

\section{Applications to other founders}

Here, we successfully developed 1,014 SNP markers spanning the whole genome, which were optimized for the definition of haplotypes of 'Fuji', and we detected QTLs by testing the effects of two haplotypes of 'Fuji'. However, we did not detect the QTLs on chr3, chr10, and chr15 for harvest day reported in previous studies (Kenis et al. 2008, Kunihisa et al. 2014, Liebhard et al. 2003b), or the major QTL for acidity on chr16, which is well-known as $M a$ (Liebhard et al. 2003b). The nondetection of these QTLs indicated the possibility that they are functionally homozygous in 'Fuji', and make no phenotypic segregation. In case that the superior characteristics of 'Fuji' are owing to the homozygosity of responsible genes, e.g. the low level of ethylene production of 'Fuji' regulated by the homozygosity of genotype ACS1-2 of 1-aminocyclopropane-1-carboxylate synthase gene (Harada et al. 2000), the QTL would not be detected by the analysis based on 'Fuji' haplotypes. In this study, we used only an average of $27 \%$ of the genotypic information defined to be in common with 'Fuji' for ANOVA. Almost all apples bred in Japan originate from only eight founders: 'Ralls Janet', 'Delicious' strains, 'Golden Delicious', 'Jonathan', 'Worcester Pearmain', 'Indo', 'McIntosh' strains, and 'Cox's Orange Pippin'. If we could define the haplotypes of remaining $73 \%$ of genome derived from these other founders and evaluate their effects, more QTLs would be identified. Recently, the Infinium array system with many more SNP markers has been developed in Rosaceae crops: $6 \mathrm{~K}$ array for cherry (Peace et al. 2012), $9 \mathrm{~K}$ for peach (Verde et al. 2012), and $8 \mathrm{~K}$ and $20 \mathrm{~K}$ for apple (Bianco et al. 2014, Chagné et al. 2012). In arrays for apple, multiple SNPs (4.7-7.7 SNPs in average) within 5-50 kbp are selected as a SNP cluster, a so-called "focal point"; the clusters are arranged at an interval of $1 \mathrm{cM}$. These focal points could define the haplotypes of broad founders, by combining multiple SNPs. The additional definition of haplotypes of other founders by using these arrays would lead to the detection of more QTLs and the estimation of the effect of each haplotype of founders, which is valuable for the prediction of phenotype in marker-assisted breeding.

Recently, PBA has been introduced to plant genetics, where the flow of haplotypes from founders to the descendants is considered for QTL detection. Bink et al. (2008, 2014) developed FlexQTL software for Bayesian QTL mapping of pedigreed families, with the assumption that each QTL is biallelic; they applied the software to fruit firmness in apple. This software was also applied to QTL mapping of eight commercially important traits of peach (FresnedoRamírez et al. 2015). Because the apple cultivars bred in 
Japan mostly originate from only eight founders, we expected that they would comprise a comparatively simple haplotype pool. Therefore, we applied a simpler method based on ANOVA for the PBA. An improved method that would enable us to evaluate each QTL as multi-allelic is desired.

\section{Acknowledgments}

We would like to sincerely thank Dr. Toshio Yamamoto and Dr. Jun-ichi Yonemaru from the National Institute of Agrobiological Sciences for their valuable advice for the planning of this study. We also would like to express our gratitude to breeding section of following institutes for kindly providing plant materials of domestic cultivars; Akita FruitTree Experiment Station, Apple Research Institute of the Aomori Prefectural Industrial Technology Research Center, Fukushima Agricultural Technology Center, Gunma Agricultural Technology Center, Hokkaido Research Organization Agriculture Research Department, Ishikawa Agriculture and Forestry Research Center, Iwate Agricultural Research Center, Miyagi Prefectural Institute of Agriculture and Horticulture, Nagano Fruit Tree Experiment Station and Yamagata Prefectural Horticultural Experiment Station. This study was supported by grants from the Ministry of Agriculture, Forestry, and Fisheries of Japan for "Genomicsbased Technology for Agricultural Improvement, NGB2007".

\section{Literature Cited}

Antanaviciute, L., F. Fernández-Fernández, J. Jansen, E. Banchi, K.M. Evans, R.Viola, R. Velasco, J.M.Dunwell, M. Troggio and D.J. Sargent (2012) Development of a dense SNP-based linkage map of an apple rootstock progeny using the Malus Infinium whole genome genotyping array. BMC Genetics 13: 203-211.

Aomori Prefectural Apple and Fruits Division (2014) Guidelines for apple production, 2014 edn. Aomori Apple Association, Aomori, p. 159.

Bianco, L., A. Cestaro, D.J. Sargent, E. Banchi, S. Derdak, M.D. Guardo, S. Salvi, J.Jansen, R. Viola, I. Gut et al. (2014) Development and Validation of a $20 \mathrm{~K}$ Single Nucleotide Polymorphism (SNP) Whole genome genotyping array for apple (Malus $\times$ domestica Borkh). PLoS ONE 9: e110377.

Bink, M.C.A.M., M.P.Boer, C.J.F.ter Braak, J.Jansen, R.E. Voorrips and W.E. van de Weg (2008) Bayesian analysis of complex traits in pedigreed plant populations. Euphytica 161: 85-96.

Bink, M.C.A.M., L.R. Totir, C.J.F. ter Braak, C.R.Winkler, M.P. Boer and O.S. Smith (2012) QTL linkage analysis of connected populations using ancestral marker and pedigree information. Theor. Appl. Genet. 124: 1097-1113.

Bink, M.C.A.M., J.Jansen, M. Madduri， R.E. Voorrips， C.E. Durel, A.B.Kouassi, F.Laurens, F. Mathis, C.Gessler, D. Gobbin et al. (2014) Bayesian QTL analyses using pedigreed families of an outcrossing species, with application to fruit firmness in apple. Theor. Appl. Genet. 127: 1073-1090.

Bowen, J.H. and C.B.Watkins (1997) Fruit maturity, carbohydrate and mineral content relationships with watercore in 'Fuji' apples. Postharvest Biol. Technol. 11: 31-38.
Cappellin, L., B. Farneti, M. Di Guardo, N. Busatto, I. Khomenko, A. Romano, R. Velasco, G. Costa, F. Biasioli and F. Costa (2015) QTL analysis coupled with PTR-ToF-MS and candidate genebased association mapping validate the role of Md-AAT1 as a major gene in the control of flavor in apple fruit. Plant Mol. Biol. Rep. 33: 239-252.

Chagné, D., R.N. Crowhurst, M. Troggio, M.W. Davey, B. Gilmore, C. Lawley, S. Vanderzande, R.P. Hellens, S. Kumar, A. Cestaro et al. (2012) Genome-wide SNP detection, validation, and development of an 8 K SNP array for apple. PLoS ONE 7: e31745.

Costa, F., W.E. Van de Weg, S. Stella, L. Dondini, D. Pratesi, S. Musacchi and S. Sansavini (2008) Map position and functional allelic diversity of $M d-\operatorname{Exp} 7$, a new putative expansin gene associated with fruit softening in apple (Malus $\times$ domestica Borkh.) and pear (Pyrus communis). Tree Genet. Genomes 4: 575-586.

Costa,F., C.P.Peace, S. Stella， S. Serra， S. Musacchi， M. Bazzani, S. Sansavini and W.E. Van de Weg (2010) QTL dynamics for fruit firmness and softening around an ethylene-dependent polygalacturonase gene in apple (Malus $\times$ domestica Borkh.). J. Exp. Bot. 61: 3029-3039.

DePristo, M., E. Banks, R. Poplin, K. Garimella, J.Maguire, C. Hartl, A. Philippakis, G. del Angel, M.A. Rivas, M.Hanna et al. (2011) A framework for variation discovery and genotyping using nextgeneration DNA sequencing data. Nat. Genet. 43: 491-498.

FAOSTAT (Food and Agriculture Organization of the United Nations) (2013) Production of apples in the world, http://faostat3.fao.org (accessed on 2015/11/04).

Fresnedo-Ramírez, J., M.C.A.M. Bink, E.van de Weg, T.R. Famula, C.H.Crisosto, T.J.Frett, K.Gasic, C.P.peace and T.M.Gradziel (2015) QTL mapping of pomological traits in peach and related species breeding germplasm. Mol. Breed. 35: 166.

Gao, Z., S. Jayanty, R. Beaudry and W. Loescher (2005) Sorbitol transporter expression in apple sink tissues: implications for fruit sugar accumulation and watercore development. J. Am. Soc. Hortic. Sci. 130: 261-268.

Harada, T., T.Sunako, Y.Wakasa, J. Soejima, T. Satoh and M.Niizeki (2000) An allele of the 1-aminocyclopropane-1-carboxylate synthase gene (Md-ACS1) accounts for the low level of ethylene production in climacteric fruits of some apple cultivars. Theor. Appl. Genet. 101: 742-746.

Harker,F.R. and I.C.Hallett (1992) Physiological changes associated with development of mealiness of apple fruit during cool storage. HortScience 27: 1291-1294.

Horigome, M., T.Nakajyou, K.Abe, M.Kotouta, H. Iwanami and S. Moriya (2010) A new cultivar 'Ozenokurenai' of apple. Bull. Gunma Agricul. Tech. Cent. 7: 45-50.

Ito, A., S. Fujine, K. Onoda, H. Sasaki, H. Tamura, S. Suzuki, A. Miura, M. Kume, S. Obara and M. Sasaki (1994) New apple cultivar 'Kio'. Bull. Iwate Hort. Exp. Sta. 7: 111-116.

Iwanami,H., S. Moriya, N. Kotoda, S. Takahashi and K.Abe (2005) Influence of mealiness on the firmness of apples after harvest. HortScience 40: 2091-2095.

Kenis, K., J.Keulemans and M.W.Davey (2008) Identification and stability of QTLs for fruit quality traits in apple. Tree Genet. Genomes 4: 647-661.

Komatsu,H., A.Usuda, T.Haniuda, H.Koike, H.Yamashita and T. Miyazawa (1998) 'Shinano Sweet', a new apple cultivar. Bull. Nagano Fruit Tree Exp. Sta. 5: 9-15.

Kon, T., T.Kudo, T.Fukasawa-Akada, S. Goto, T.Akahira, I. Machida, M. Shirakawa and N.Akita (2012) New apple cultivars, 'Aori 9', 'Aori 13' and 'Aori 15'. Bull. Apple Res. Inst. Aomori Pref. Ind. 
Tec. Res. Cent. 36: 45-72.

Kumar, S., D.J.Garrick, M.C.Bink, C. Whitworth, D. Chagné and R.K. Volz (2013) Novel genomic approaches unravel genetic architecture of complex traits in apple. BMC Genomics 14: 393.

Kunihisa, M., S. Moriya, K.Abe, K. Okada, T. Haji, T. Hayashi, H. Kim, C. Nishitani, S. Terakami and T. Yamamoto (2014) Identification of QTLs for fruit quality traits in Japanese apples: QTLs for early ripening are tightly related to preharvest fruit drop. Breed. Sci. 64: 240-251.

Li,H. and R. Durbin (2009) Fast and accurate short read alignment with Burrows-Wheeler Transform. Bioinformatics 25: 1754-1760.

Li, H. (2011) A statistical framework for SNP calling, mutation discovery, association mapping and population genetical parameter estimation from sequencing data. Bioinformatics 27: 2987-2993.

Liebhard, R., B. Koller, L. Gianfranceschi and C. Gessler (2003a) Creating a saturated reference map for the apple (Malus $\times$ domestica Borkh.) genome. Theor. Appl. Genet. 106: 1497-1508.

Liebhard, R., M. Kellerhals, W. Pfammatter, M. Jertmini and C. Gessler (2003b) Mapping quantitative physiological traits in apple (Malus $\times$ domestica Borkh.). Plant Mol. Biol. 52: 511-526.

Longhi, S., M. Moretto, R. Viola, R. Velasco and F. Costa (2012) Comprehensive QTL mapping survey dissects the complex fruit texture physiology in apple (Malus $\times$ domestica Borkh.). J. Exp. Bot. 63 : 1107-1121.

MAFF (Ministry of Agriculture, Forestry and Fisheries) (2012) The list of the apple production of each cultivar. http: //www.maff.go. $\mathrm{jp} / \mathrm{j} /$ tokei/kouhyou/tokusan kazyu/ (accessed on 2015/11/04).

MAFF (Ministry of Agriculture, Forestry and Fisheries) (2014) The list of the planted area, yield (10a), total yield and shipping volume of each fruits. http: //www.maff.go.jp/j/tokei/kouhyou/sakumotu/ sakkyou_kazyu/index.html (accessed on 2015/11/04).

Marlow, G.C. and W.H.Loescher (1984) Watercore. Hortic. Rev. 6: 189-251.

Martin, M. (2011) Cutadapt removes adapter sequences from highthroughput sequencing reads. EMBnet.Journal 17: 10-12.

Moriya, S., H. Iwanami, N. Kotoda, S. Takahashi, T. Yamamoto and K.Abe (2009) Development of a marker-assisted selection system for columnar growth habit in apple breeding. J. Japan. Soc. Hort. Sci. 78: 279-287.

Nakajo, T. (1990) New apple cultivar 'Sinsekai'. Gunma J. Agricul. Res. D, Horticulture 5: 35-42.

Nakajo,T. and M.Horigome (1992) New apple cultivar 'Gunma meigetu'. Gunma J. Agricul. Res. 7: 29-33.

Nakajo, T. and M.Horigome (1995a) A New apple cultivar 'Honey Queen'. Res. Bull. Gunma Hort. Exp. Sta. 1: 21-25.

Nakajo, T. and M. Horigome (1995b) A new apple cultivar 'Slim Red'. Res. Bull. Gunma Hort. Exp. Sta. 1: 13-19.

Nakano, S., H. Tsugawa, S. Yamada and S. Nobatake (2003) Breeding of the new apple variety 'Syusei' with excellent characteristics. Bull. Ishikawa Agricul. Res. Cent. 25: 9-12.

Niino, K., M. Ishiguro, Y.Abiko, K. Nishimura and Y.Honma (2009) New apple cultivar 'Fast Lady'. Issu. Yamagata pref. Agricul. Res. 1: 49-59.

Nobile,P.M., F.Wattebled, V.Quecini, C.L.Girardi, M.Lormeau and F. Laurens (2011) Identification of a novel $\alpha$-L-arabinofuranosidase gene associated with mealiness in apple. J. Exp. Bot. 62: 43094321.

Okudaira, M., S. Suzuki and K. Onoda (2003) New apple cultivar ripens in late September, Iwate No. 6. Tohoku Agricul. Res. 56: 143 144.

Onoue, N., M. Yamada, T. Yamamoto, S. Terakami, C. Nishitani,
M. Kunihisa, N. Takada, S. Nishio, Y. Sawamura and T. Saito (2015) Kinship and inbreeding estimates based on microsatellite markers in breeding of Japanese pear (Pyrus pyrifolia Nakai). Euphytica 205: 539-555.

O'Rourke, D. (2003) World production, trade, consumption and economic outlook for apples. In: Ferree, D.C. and I.J. Warrington (eds.) Apples: botany, production and uses, CAB International, Oxon, pp. $15-29$.

Peace, C., N. Bassil, D.Main, S. Ficklin, U.R.Rosyara, T. Stegmeir, A. Sebolt, B. Gilmore, C. Lawley, T.C. Mockler et al. (2012) Development and evaluation of a genome-wide $6 \mathrm{~K} \mathrm{SNP}$ array for diploid sweet cherry and tetraploid sour cherry. PLoS ONE 7: e48305.

Sadamori, S., Y. Yoshida, H.Murakami and S. Ishizuka (1963) New apple variety 'Fuji'. Bull. Hort. Res. Sta. Series C 1: 1-6.

Sakuma, N., T. Kunisawa, H. Yamaga and H. Matsuno (1999) New apple cultivar 'Hoozuri'. Bull. Fukushima Fruit Tree Exp. Sta. 17: $7-11$.

Segonne, S.M., M. Bruneau, J.M.Celton, S.L. Gall, M. Francin-Allami, M. Juchaux, F. Laurens, M. Orsel and J.P. Renou (2014) Multiscale investigation of mealiness in apple: an atypical role for a pectin methylesterase during fruit maturation. BMC Plant Biol. 14: 375392.

Silfverberg-Dilworth,E., C.L.Matasci, W.E. Van de Weg, M.P.W. Van Kaauwen, M. Walser, L.P. Kodde, V. Soglio, L. Gianfranceschi, C.E. Durel, F. Costa et al. (2006) Microsatellite markers spanning the apple (Malus $\times$ domestica Borkh.) genome. Tree Genet. Genomes. 2: 202-224.

Soejima, J., Y.Yoshida, T.Haniuda, H.Bessho, T. Masuda, S. Komori, S. Tsuchiya, Y.Ito, T. Sanada, K.Abe et al. (2012a) New apple cultivar 'Kitaro'. Bull. Natl. Inst. Fruit Tree Sci. 13: 27-38.

Soejima, J., H. Bessho, Y.Yoshida, T. Haniuda, T. Masuda, S. Komori, S. Tsuchiya, Y. Ito, T. Sanada, K. Abe et al. (2012b) New apple cultivar 'Kotaro'. Bull. Natl. Insti. Fruit Tree Sci. 14: 11-23.

Tanba, J., S.Tanno, H.Sato, T.Niizuma, M.Kagaya and T.Taguchi (1994) The new apple cultivar-'Akita Gold'. Bull. Akita FruitTree Exp. Sta. 24: 1-12.

Tanno, S., T. Taguchi, J.Tanba, H. Suzuki and K.Kon (1980) A new variety of apple, 'Sensyu'. Bull. Akita Fruit-Tree Exp. Sta. 12: $1-12$.

Ueta, J., M. Terui, K. Kobayashi, H. Sato and I. Takahashi (2011) New apple cultivar 'Akitabeniakari'. Bull. Akita Fruit-Tree Exp. Sta. 29: $1-7$.

van Ooijen, J.W. (2006) JoinMap 4 Software for the calculation of genetic linkage maps in experimental populations. Kyazma B.V., Wageningen, the Netherlands.

Velasco, R., A.Zharkikh, J.Affourtit, A.Dhingra, A.Cestaro, A Kalyanaraman, P.Fontana, S.K. Bhatnagar, M. Troggio, D. Pruss et al. (2010) The genome of the domesticated apple (Malus $\times$ domestica Borkh.). Nat. Genet. 42: 833-839.

Verde, I., N.Bassil, S. Scalabrin, B. Gilmore, C.T.Lawley, K. Gasic, D. Micheletti, U.R. Rosyara, F.Cattonaro, E. Vendramin et al. (2012) Development and evaluation of a 9 K SNP array for peach by internationally coordinated SNP detection and validation in breeding germplasm. PLoS ONE 7: e35668.

Voorrips, R.E., M.C.A.M. Bink and W.E. van de Weg (2012) Pedimap: Software for the visualization of genetic and phenotypic data in pedigrees. J. Hered. 103: 903-907.

Williams, E.B.， J. Janick， F.H.Emerson， D.F. Dayton， J.B. Mowry, L.F.Hough and C.Bailey (1972) 'Priscilla', a fall red apple with resistance to apple scab. Fruit Varieties 26: 34-35.

Yamada, M., C.Suzuki, M.Ishida, H.Kitayama and T. Sato (1989) 
Apple cultivar, 'Sekaiichi'. Bull. Aomori Apple Exp. Sta. 25: 6172.

Yamamoto,T., H. Nagasaki, J.Yonemaru, K.Ebana, M.Nakajima, T. Shibaya and M. Yano (2010) Fine definition of the pedigree haplotypes of closely related rice cultivars by means of genome-wide discovery of single-nucleotide polymorphisms. BMC Genomics 11: 267-280.

Yoshida, M., H.Muramatsu, T.Minegishi, R. Ogano, H.Watanabe, M. Kakizaki, S. Tanaka, Y. Inagawa and T. Noda (2002) A new apple variety 'Maoi'. Bull. Hokkaido Pref. Agricul. Exp. Sta. A82: 41-48.

Yoshida, Y., T. Haniuda, S. Tsuchiya, T. Sanada and S. Sadamori (1982) New apple cultivar 'Kitakami'. Bull. Fruit Tree Res. Sta. Ser. C,
Morioka 9: 1-13.

Yoshida, Y., T.Haniuda, S. Tsuchiya, T. Sanada, T.Masuda, H. Bessho and S. Sadamori (1985a) New apple cultivar 'Himekami'. Bull. Fruit Tree Res. Sta. Ser. C, Morioka 12: 1-10.

Yoshida, Y., T.Haniuda, S. Tsuchiya, T. Sanada, T. Masuda, H. Bessho and S. Sadamori (1985b) New apple cultivar 'Iwakami'. Bull. Fruit Tree Res. Sta. Ser. C, Morioka 12: 11-20.

Yoshida, Y., T. Haniuda, S. Tsuchiya, T. Sanada, T.Masuda, H. Bessho and D.W.Mckenzie (1988) New apple cultivar 'Sansa'. Bull. Fruit Tree Res. Sta. Ser. C, Morioka 15: 1-12.

Yoshida, Y., T. Haniuda, S. Tsuchiya, T. Masuda, H. Bessho, T. Sanada, S.Komori and D.W.McKenzie (1991) New apple cultivar 'Kizashi'. Bull. Nati. Insti. Fruit Tree Sci. 20: 25-40. 